\title{
Treatment preferences in fibromyalgia patients: A cross-sectional web-based survey
}

\author{
Elia Valentini (iD) | Eleonora Fetter (iD) | Sheina Orbell ${ }^{(1)}$
}

Department of Psychology and Centre for Brain Science, University of Essex, Colchester, UK

\section{Correspondence}

Elia Valentini, Centre for Brain Science, Department of Psychology, University of Essex, Wivenhoe Park, Colchester CO4 3SQ, UK.

Email: evalent@essex.ac.uk

\begin{abstract}
Background: Therapeutic approaches to fibromyalgia (FM) are shifting towards a combined multi-treatment approach to tackle the variety of symptoms experienced in FM. Importantly, little is known about FM patients' attitude towards the available treatments.
\end{abstract}

Methods: A cross-sectional web survey obtained responses from 464 individuals who satisfied diagnostic criteria for FM. Respondents were asked to report which treatments they adopted in their past, present and intend to adopt in the future. They also rated their level of well-being, depression, anxiety and pain catastrophizing.

Results: Data revealed a predominantly combined multi-treatment approach in a sample characterized by middle-aged, Caucasian women. Respondents reported pervasive use of pharmacological therapy but had also adopted non-pharmacological treatment in the past. Future intentions clustered on alternative treatment or no treatment. Regression analyses revealed that pharmacological treatment in the past was predictive of both pharmacological and non-pharmacological treatments in the present. However, use of non-pharmacological treatment in the past was uniquely predictive of its reuse in the present and future. This pattern was also accounted for by individual differences in pain magnification and well-being in the past.

Conclusions: Treatment preferences of FM individuals reveal an ambivalent combination of heavy reliance on pharmacological treatment with lower but consistent reliance on non-pharmacological treatment for those individuals who used it in the past and present. This finding may inform longitudinal research into the relationship between pharmacological and non-pharmacological treatment preference in FM patients, which could in turn inform guidelines for individualized therapeutic plans for clinicians.

Significance: Individuals with fibromyalgia reported the use of non-pharmacological and pharmacological treatments in the past but a predominant use of a pharmacological approach overall. Patterns of treatment experienced in the past were differentially related to future preferences. Pharmacological treatment in the past was likely to lead to both pharmacological and non-pharmacological choices in the present. However, non-pharmacological treatment in the past was more likely to be chosen again in the present and future, but unlikely to lead to a pharmacological choice. 


\section{1 | INTRODUCTION}

According to the recent classification of chronic pain for the International Classification of Diseases, fibromyalgia (FM) is conceived as a "chronic primary pain," namely a condition whereby widespread pain should be considered as the disease per se rather than as a symptom occurring in the context of an unrelated condition (Treede et al., 2019). Such diagnostic coding reflects the debilitating dimension of FM, a disorder characterized by core symptoms such as persistent and intense muscle and joint pain, sleep disturbances and fatigue (Wolfe et al., 2010). Over the years, clinicians have attributed several additional symptoms to FM including cognitive dysfunctions, depression, anxiety and sensory disturbances (Wolfe et al., 2016). Estimates of the prevalence of FM range between $0.5 \%$ and $5 \%$ of the total population (Bernardy, Füber, Köllner, \& Häuser, 2010; Branco et al., 2010; Jones et al., 2015). A more recent meta-analysis of epidemiological evidence suggests that $1.78 \%$ of the general population, and $3.98 \%$ of the female population, have been diagnosed with FM (Heidari, Afshari, \& Moosazadeh, 2017).

Treatment outcome in FM is often centred on pain reduction; however, the IMMPACT survey showed that psychological well-being is a crucial dimension to consider when assessing treatment effectiveness in people with chronic pain (Turk et al., 2008). There is evidence that both pharmacological and non-pharmacological treatments lead to clinically meaningful improvement in FM patients (Cording, Moore, Derry, \& Wiffen, 2015; Häuser, Urrútia, Tort, Üçeyler, \& Walitt, 2013; Uceyler, Hauser, \& Sommer, 2011). The most recent European League Against Rheumatism (EULAR) recommendations indicate strong evidence for a beneficial effect of physical exercise and suggest patient education and non-pharmacological therapies as initial treatment strategies and for ongoing management of severe cases (Macfarlane et al., 2017). These conclusions were supported by several findings, including large surveys on the US and German populations affected by FM (Bennett, Jones, Turk, Russell, \& Matallana, 2007; Häuser et al., 2012). However, clinicians recognize that FM is a multifactorial condition with heterogeneous configuration across individuals (Rooij et al., 2013; Turk, Okifuji, Sinclair, \& Starz, 1998), thus requiring a biopsychosocial, multidisciplinary, personalized approach to be treated effectively (Häuser, Perrot, Clauw, \& Fitzcharles, 2017).

Randomized controlled trials have shown that multiple non-pharmacological treatments, such as psychotherapy, exercise therapy, education and physiotherapy, are effective in reduction of FM symptoms. Importantly, cognitive behavioural therapy (CBT) ostensibly reduces several FM symptoms, including pain at follow-up (Bernardy, Klose, Welsch, \& Häuser, 2018). Despite this evidence, the most prominent treatment strategy for the reduction of FM symptoms continues to be pharmacological. And yet, past surveys showed that patients report little satisfaction with treatment, including pharmacological therapies (Lauche et al., 2013; Robinson et al., 2013). Therefore, therapeutic approaches have been shifting towards a multi-component approach to treat the condition (Teo, Mohan, \& Oelke, 2017). To date, however, there is little evidence that even a multi-component approach is effective in substantially reducing FM symptoms. For example, Van De Houte et al. (2017) recently observed that only a fourth to a third of FM patients assessed in the context of a multi-component treatment program had improvement in treatment outcomes.

The aim of the present internet-based cross-sectional survey was to investigate patterns of use of different available treatments in the past and present among people with FM, and their intention to use different treatments in the future. They also reported their well-being across the past, present and the future. In addition, we investigated the relationship of well-being, depression, anxiety and pain catastrophizing with attitude towards treatment.

\section{MATERIALS AND METHODS}

\section{1 | Respondents}

We contacted several FM support groups/organizations via e-mail and social media between July and August 2017 (organizations' names and members' nationality are reported in Supporting Information S1). Members of these associations $(N=666)$ voluntarily completed an internet-based crosssectional survey (developed using Qualtrics, Provo, UT). Respondents were informed that the aim of the survey was to collect information about their condition and to evaluate the treatments they felt were most successful in reducing the impact of their symptoms. Also, how likely they were to use the treatments in the future. They were informed that the survey would take approximately $20 \mathrm{~min}$ to complete. Respondents gave their informed consent before beginning the study, which was approved by the University of Essex ethics committee (project code EV1501).

\subsection{Survey content}

The complete survey consisted of five blocks: information and consent, demographics and information concerning diagnosis, treatment choice, treatment rating, personality and well-being questionnaires.

\subsection{1 | FM assessment}

The main criterion for participation was a diagnosis of FM. Participants were asked "How long has it been since you were 
diagnosed with FM?" (Table 1). We first asked respondents to provide us with some demographic information. They then completed the symptom severity (SS) and widespread pain index (WPI) to assess the impact of the symptoms and generalized pain (Wolfe et al., 2010). Responses to these measures were used to screen participants for inclusion in analyses.

\subsection{2 | Mood, pain catastrophizing and well- being}

Participants completed the patient health questionnaire (PHQ-9; Kroenke \& Spitzer, 2002) to investigate the current

TA B L E 1 Demographics and disease-related questions $(N=464)$

$\begin{array}{lll}\text { Sample feature } & \begin{array}{l}\text { N or } \\ \text { Median }^{\text {a }}\end{array} & \text { \% or range } \\ \text { Gender } & 28 & 6 \\ \quad \text { Male } & 436 & 94 \\ \text { Female } & 49 & 69 \\ \text { Age } & & \\ \text { Ethnicity } & 418 & 90.1 \\ \text { Caucasian } & 4 & 0.9 \\ \text { Black or African American } & 6 & 1.3 \\ \text { Asian/ Pacific Islander } & 1 & 0.2 \\ \text { Hispanic or Latino } & 35 & 7.5 \\ \text { Other } & & \end{array}$

Time from diagnosis

Less than 2 years

Less than 5 years 179

38.6

5 years or more

89

19.2

More than 10 years

90

19.4

Illness intensity rating

$\begin{array}{lll}\text { Severe } & 206 & 44.4 \\ \text { Moderate } & 236 & 50.9 \\ \text { Mild } & 22 & 4.7\end{array}$

Symptoms from diagnosis

$\begin{array}{lll}\text { Pain } & 464 & 100\end{array}$

$\begin{array}{lll}\text { Depression } & 370 & 79.7\end{array}$

$\begin{array}{lll}\text { Sleep disturbances } & 449 & 96.8\end{array}$

$\begin{array}{lll}\text { Anxiety } & 379 & 81.7\end{array}$

Fatigue $\quad 460 \quad 99.1$

Cognitive dysfunctions $\quad 425 \quad 91.6$

WPI score $\quad 12 \quad 16$

$\begin{array}{lll}\text { SS score } & 9 & 12\end{array}$

Abbreviations: FM, fibromyalgia; SS, symptom severity score; WPI, widespread pain index.

${ }^{\mathrm{a}}$ No missing values. disposition to depressive symptoms, and the State-Trait Anxiety Inventory (STAI; Spielberger et al., 1983) to assess anxiety. The PHQ-9 partitions overall individual scores into five categories: (a) minimal symptoms; (b) minor depression, dysthymia; (c) major depression, mild; (d) major depression, moderately severe; (e) major depression, severe. In addition, respondents completed the pain catastrophizing scale (PCS; Sullivan, Bishop, \& Pivik, 1995), a questionnaire that measures the cognitive/emotional attitude towards pain. Lastly, they completed the Temporal Satisfaction with Life (TSL) scale (Pavot, Diener, \& Suh, 1998) to quantify their wellbeing in the past, present and future.

\subsection{3 | Treatment preferences}

Participants were presented with a list of pharmacological treatments, psychological interventions, physical therapy, physiotherapy and occupational therapy. Participants were asked to indicate which they had used in the past ("Since your diagnosis did you have any of these treatments?"), present ("Since your diagnosis are you still having any of these treatments?") and what they wished to use in the future ("Are you planning or thinking of using these treatments in the future?").

\section{3 | Data analysis approach}

Data entry was checked for accuracy. Data analysis was performed in SPSS Statistics (IBM SPSS Statistics for Windows, version 22.0, IBM Corp). None of the variables were normally distributed. All statistics were two tailed at the 0.05 significance level and missing values were dealt with casewise deletion. Bootstrap or Monte Carlo approximation with confidence level was calculated for each of the inferential statistics (10,000 samples).

\subsection{1 | Single versus multiple combined treatment}

Respondents could select their treatments among several options: pharmacological treatments (i.e. a series of drugs most frequently applied to treatment of FM symptoms), psychological interventions (i.e. a few representative psychological treatments), physical therapy, physiotherapy and occupational therapy. Moreover, respondents were given the option to specify alternative treatments (e.g. naturopathy and acupuncture) that were not mentioned in this list, or no treatment at all. Each respondent could provide more than one response, thus permitting assessment of the difference within and between respondents in terms of multiple combined treatment 
versus single treatment across time. We calculated Friedman ANOVA first to assess the presence of difference in choices across time for all the treatment categories. Significant differences were then further analysed using Wilcoxon matched pairs test.

\subsection{2 | Mapping the relationship between treatment preference and time frame of the choice}

Having no specific hypotheses about the direction of the relationship between treatment choice and time frame of the choice, we explored this relationship by applying correspondence analysis (CA). CA is a dimensionality reduction technique that allows us to describe the relationship between categorical data (Sourial et al., 2010). CA can also be conceived as a pattern recognition method that treats nominal data sets in rows and columns within two-way tables as points on a multidimensional graphical map, also called a biplot (Benzecri, 1992). Akin to factor analysis or principal component analysis, CA aims to explain the overall inertia (i.e. variance) in the model with the least number of dimensions. Rows with similar frequencies will have points that are close together in the bidimensional space. The same reasoning applies to columns. The objective of this analysis is to show the co-occurrence of the categories in a bidimensional space where the proximity of these categories may indicate meaningful associations among themselves. We expressed the inertia using the chi-squared statistic (i.e. weighted Euclidean distance) that indexes the distance between points on the biplot (Greenacre, 2013). We used a symmetrical normalization method for a two-dimensional model explaining the inertia of raw points. It is noteworthy that the index of distance makes it possible to interpret the associations between categories of the same variable (e.g. between different treatments) but not between variables of different categories (e.g. between different treatments and temporal frames). However, it is possible to make interpretations of the general locations of row and column points, and their relations within each type of point.

\subsubsection{Assessing the predictive role of treatment preference over time}

We performed multinomial logistic regression to discover whether the use of a non-pharmacological or pharmacological approach in the past was predictive of present and future treatment preference. We separately analysed whether past treatment preference was predictive of present and future treatment preference. Therefore, three categories entered the analysis: pharmacological, non-pharmacological and both. We selected the "both" category as reference baseline as this was the most common approach in the past. Results are reported as relative log odds and $S E$.

\subsubsection{Well-being and other individual differences}

We analysed the difference in well-being scores across time using Friedman ANOVA. Significant differences were then further analysed using Wilcoxon matched pairs test. We used again multinomial logistic regression to explore whether any of the mood, anxiety, pain catastrophizing and well-being scores were predictive of the use of non-pharmacological or pharmacological treatment in the past. We then re-instated the models looking at the effect of (a) past on present and (b) present on future treatment preference by adding the mood, anxiety, pain catastrophizing and well-being scores as covariates in separate regression analyses. Results are reported as relative $\log$ odds and $S E$.

\section{3 | RESULTS}

\section{1 | Sample demographics and FM symptoms}

The sociodemographic and disease profile of the final sample are reported in Table 1. Respondents who did not meet the inclusion criterion of WPI $>7$ and SS $>5$ or WPI 3-6 and SS $>9$ were excluded from the data analysis as they were not consistently clinically classifiable as FM patients. This operation led to us excluding the data from 149 respondents who did not fully complete the WPI or SS and 53 respondents whose scores did not meet the inclusion criteria from the whole data set of 666 respondents, thus leaving us with a sample of 464 respondents $(69.7 \%)$. These were mostly white Caucasians $(90.1 \%)$ with a median age of 49 years (ranging between 16 and 85). An overwhelming majority of respondents were female (94\%) with a mild bimodal pattern of diagnosis onset, being largely distributed in either a recent diagnosis $(38.6 \%)$ or a long-standing condition $(22.8 \%)$. A similar bimodal distribution was applied to their rating of illness intensity, showing that the respondents were split between a moderate and severe illness (50.9\% and $44.4 \%)$. Pain and fatigue were the most represented symptoms (100\% and 99.1\%). Nevertheless all the symptoms were highly represented in the sample. Both the median WPI and SS scores are high as expected in individuals diagnosed with FM even though there was a large variability in both scale scores. 


\section{2 | Single or multiple combined treatment?}

The treatment choice differed across time $\left(\chi_{434}^{2}=200.48\right.$; $p<.001)$. Figure 1 shows how this difference was explained by higher adoption of combined treatment against single treatment (either pharmacological or non-pharmacological) in the past versus present $\left(Z_{443}=-13.25 ; p<.001\right)$ and in the future prospect versus present $\left(Z_{434}=-6.89 ; p<.001\right)$. However, less combined treatment was intended in the future compared to the past $\left(Z_{434}=-6.34 ; p<.001\right)$.

Figure 2 describes the respondents' choice of treatments in the past, present and future, while (Supporting Information S2: Table S1) provides more detail about the frequency with which specific pharmacological and psychological treatments have been sought. The most salient information concerns the amount of people adopting pharmacological treatment in the past (64.9\%), present (55\%) and planning to adopt it in the future $(32.8 \%)$ compared to other treatments.

\section{3 | Map of the relationship between treatment preference and time frame of reference}

We applied CA to explore the structure of the relationship between the different type of treatments and the preference attached to them in each of the temporal frames. The graphical representation (Figure 3) summarizes these relationships. As guidance for interpretation, the greater the distance between a type of treatment and a temporal frame the less likely is they are associated in accounting for the data. The CA model was highly significant $\left(\chi_{434}^{2}=217.90 ; p<.001\right)$. The total inertia was $9.4 \%$. Of this variance, $65.5 \%$ was accounted for by Dimension 1 while $34.5 \%$ was accounted for by Dimension

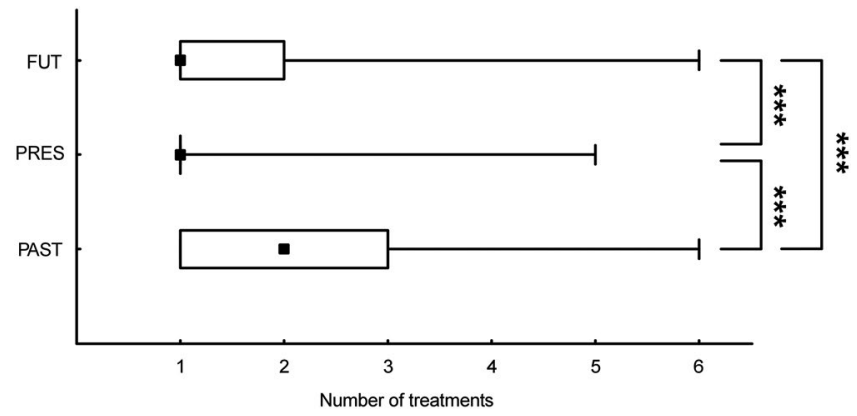

F I G U RE 1 Box-plots characterizing the relationship between temporal frame ( $y$ axis) and treatment choice ( $x$ axis). The small square indicates the median, the box indicates the 25 th and 75 th percentiles and the whiskers indicate the extreme values. Note how most of the respondents used at least two different treatments in the past. Significant differences are indicated with asterisks $(* * * p<.001)$. Abbreviations: FUT, Future; PRES, Present

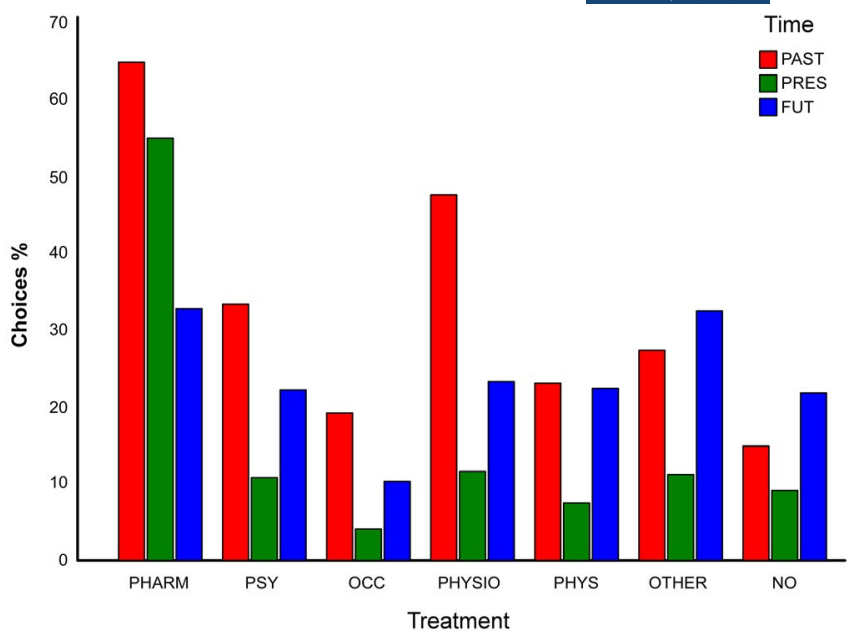

F I G URE 2 Bar chart detailing the per cent frequency of choice ( $y$ axis) per each treatment category ( $x$ axis) in the past (black), present (grey) and future (lighter grey). Note the greater preference for pharmacological treatment in the past and present as well as physiotherapy in the past. Abbreviations: FUT, Future; PRES, Present. PHARM, pharmacological; PSY, psychological; OCC, occupational; PHYSIO, physiotherapy; PHYS, physical therapy; OTHER, other treatment; NONE, no treatment

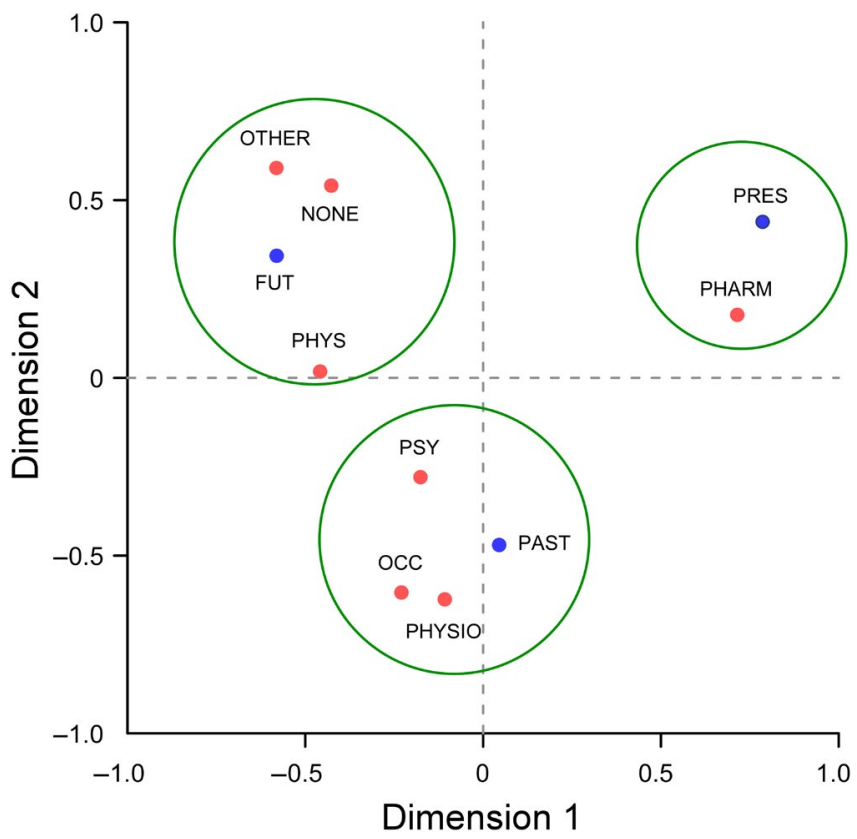

F I G U RE 3 Biplot representing the treatment choice (red) and time frame of the choice (blue). Note the three clouds clustering preference for psychological treatment, physiotherapy and occupational therapy in the past (bottom), pharmacological treatment in the present (top right) and other treatment or no treatment in the future (top left). Abbreviations: FUT, Future; PRES, Present. PHARM, pharmacological; PSY, psychological; OCC, occupational; PHYSIO, physiotherapy; PHYS, physical therapy; OTHER, other treatment; NONE, no treatment 
2. Supporting Information S3: Table S2 provides detail of row and column statistics.

Present choices (Figure 3, top right quadrant) loaded more on Dimension 1 (54.6\%) than Dimension 2 (23.4\%). Likewise, future choices (Figure 3, top left quadrant) loaded more on Dimension $1(45 \%)$ than Dimension 2 (21.7\%). On the contrary, past choices (Figure 3, bottom right quadrant) loaded more on Dimension 2 (54.8\%) than Dimension 1 (0.4\%). Overall, Dimension 1 explained $81.5 \%$ and $79.7 \%$ of present and future choices, respectively, whereas the extraction of Dimension 2 explained $98.7 \%$ of the variance in the past choices. Concerning the treatment options, Dimension 1 was best contributed by pharmacological treatment (63\%; Figure 3, top right), other treatment (17.4\%; Figure 3, top left) and physical therapy (9.0\%; Figure 3, top left) while Dimension 2 was best contributed by physiotherapy (35.7\%; Figure 3, bottom left), other treatment (24.7\%; Figure 3, top left) and no treatment (14.9\%; Figure 3, top left). Dimension 1 explained $95.7 \%$ of pharmacological treatment, $57.1 \%$ of other treatment and $99.9 \%$ of physical therapy, whereas the extraction of Dimension 2 accounted for $96 \%$ of physiotherapy, $42.9 \%$ of other treatment and $53.8 \%$ of no treatment.

The biplot displays an extreme distancing between pharmacological treatment and all the other survey options along the $x$ axis (red dots), particularly against other treatment, physical therapy and no treatment. Likewise, it indicates an opposition between past choices versus present and future choices on the $y$ axis (blue dots). The plot also reveals three main clouds where Euclidean distances are nearest. A cluster on the bottom suggests the association of past preferences with physiotherapy, psychological and occupational treatment (bottom right and left quadrant). A cluster on the top right quadrant hints to pharmacological treatment as the best treatment in representing present respondents' choices. Finally, a third cluster hints to the association between future attitude towards treatment and physical therapy, other alternative treatment or no treatment at all (top left quadrant). See Supporting Information S4 for a characterization of what alternative treatment was reported by survey respondents (Table S3).

\subsection{Is the previous or current treatment preference predictive of subsequent choices?}

Past versus Present preference. Figure 4 (left) summarizes the model's effects in terms of predicted probabilities. The model significantly explained the data $(n=312)$. Specifically, past attitude was predictive of present choices $\left(\chi_{4}^{2}=142.14\right.$ $p<.001)$. The model correctly predicted an overall $61.5 \%$ of choices. The relative log odds indicate that, compared to the "both" category, respondents using pharmacological treatment in the past were more likely to keep using it in the present ( $b=23.06 ; S E=0.56 ; p<.001$; Figure 4, left graph, blue fit, left). Conversely, those using non-pharmacological treatments in the past were equally likely to use pharmacological treatment in the present $(b=0.34 ; S E=1.78 ; p=.59$; Figure 4, left graph, red fit, left). Importantly, respondents using pharmacological treatment in the past were more likely to use non-pharmacological treatment in the present $(b=19.21 ; S E=9.15$; $p<.001$; Figure 4, left graph, blue fit, centre). Similarly, respondents using non-pharmacological treatments in the past were more likely to use it again in the present $(b=2.03$; $S E=1.65 ; p<.001 ;$ Figure 4, left graph, red fit, centre).

Present versus Future preference. Figure 4 (right) summarizes the model's effects in terms of predicted probabilities. The model significantly explained the data $(n=264$; $\left.\chi_{4}^{2}=96.12 ; p<.001\right)$. The model correctly predicted an overall $53.4 \%$ of choices. The relative $\log$ odds indicate that, compared to the "both" treatment category, respondents using pharmacological treatment in the present were equally likely to express the intention to keep using it in the future $(b=-0.36 ; S E=0.23 ; p=.10)$. Similarly, those using non-pharmacological treatments in the present were equally likely to express the intention of using pharmacological treatment in the future $(b=-0.18 ; S E=0.61 ; p=.76)$. Crucially, respondents using non-pharmacological treatments in the present were more likely to report wanting to use it again in the future $(b=2.06 ; S E=0.43 ; p<.001$; Figure 4, right graph, red fit, centre). More detailed information on the

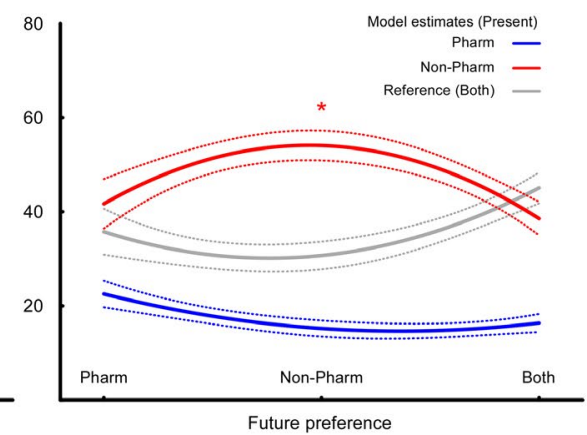

F I G U RE 4 Per cent predicted probabilities ( $y$ axis) indicating how likely, compared to the "both" treatment category (grey), respondents were to choose pharmacological (blue) or non-pharmacological (red) treatment in the present based on past choices (left graph) or as potential therapy in the future based on present choices (right). Significant predictions are indicated with asterisks $(* p<.001)$. Abbreviations: Pharm, pharmacological; Non-Pharm, non-pharmacological 
different alternative treatments is reported in Supporting Information S4: Table S3.

\section{5 | Mood, anxiety, catastrophizing and well-being estimates}

Table 2 reports the central tendency and variability in scores of mood, anxiety, pain catastrophizing and well-being. Most respondents reported a mild level of depressive symptoms (scoring 8 of 27) and a high level of state and trait anxiety (scoring 50 of 60). The PCS scores were above the average of the normative sample (i.e. $>20$; Sullivan et al., 1995). Likewise, satisfaction with life in the past, present and future (TSL) was higher than the norms obtained amongst the reference student sample (Pavot et al., 1998).

In addition, Friedman ANOVA revealed that FM respondents reported different levels of well-being over time $\left(\chi_{383}^{2}=24.34 ; p<.001\right)$. This was explained by lower well-being in the past compared to present $\left(Z_{383}=-4.46 ; p<.001\right)$ and future $\left(Z_{383}=-4.22 ; p<.001\right)$ but no difference in well-being between present and future $\left(Z_{383}=-0.29 ; p=.78\right)$.

\section{6 | Predictive role of mood, anxiety, catastrophizing and well-being estimates on treatment preference}

We replicated the regression analysis of treatment preference by introducing the mood and well-being questionnaire scores as continuous covariates in the models.

TA B L E 2 Mood, anxiety, catastrophizing and well-being questionnaire scores

\begin{tabular}{|lrr}
\hline Questionnaire & Mean & SD \\
\hline PHQ-9 & 8.53 & 4.76 \\
\hline STAI Y-1 & 50.32 & 2.39 \\
\hline STAI Y-2 & 56.26 & 8.67 \\
\hline PCS & & \\
Rumination & 11.05 & 5.71 \\
Magnification & 3.13 & 2.34 \\
Helplessness & 13.04 & 6.32 \\
Total & 25.46 & 12.31 \\
\hline TSWLS* & & \\
Past & 22.66 & 8.02 \\
Present & 24.96 & 8.24 \\
Future & 24.90 & 7.54 \\
Total & 72.52 & 18.77 \\
\hline
\end{tabular}

Abbreviations: PCS, pain catastrophizing scale; PHQ-9, patient health questionnaire (9 items); STAI Y-1 and Y-2, State-Trait Anxiety Inventory (Y-1, State; Y-2, Trait; TSWLS, Temporal Satisfaction With Life Scale) (*data for this questionnaire rely on a partial sample of 383 respondents)
Past versus Present preference. Pain catastrophizing and well-being in the past were the only covariates that had a significant effect on the regression models. The model including the three pain catastrophizing subscales $(n=312)$ significantly contributed to predict present choices $\left(\chi_{6}^{2}=137.68\right.$; $p<.001)$. The model correctly predicted an overall $62.5 \%$ of choices. The pain magnification subscale alone explained the effect, with respondents reporting higher pain magnification being more likely to choose non-pharmacological treatment in the present if they had used it already in the past $(b=0.44 ; S E=0.13 ; p=.001$; Figure 5, left graph, red fit). The model including the well-being in the past subscale $(n=281)$ significantly contributed to predict present choices $\left(\chi_{6}^{2}=183.38 ; p<.001\right)$. The model correctly predicted an overall $61.6 \%$ of choices. Respondents scoring lower in well-being in the past were more likely to use either pharmacological $(b=-0.04 ; S E=0.02 ; p=.03$; Figure 5, right graph, blue fit) or non-pharmacological treatment in the present $(b=-0.07 ; S E=0.03 ; p=.03$; Figure 5 , right graph, red fit), whereas their use in combination was linked to greater well-being (Figure 5, reference category, both, grey line).

Present versus Future preference. None of the models revealed a significant main effect.

\section{4 | DISCUSSION}

The results of our cross-sectional web survey revealed a sample consisting of middle-aged, Caucasian women (Table 1) who had used a range of different type of treatments to alleviate their FM symptoms in the past and planned to do so in the future (Figures 1 and 2; Table S1). Correspondence analysis of treatment preference across time (Figure 3; Table S2) suggested a significant diversity of choice clustering whereby non-pharmacological treatments dominated in the past (i.e. psychological treatment, physiotherapy and occupational therapy), whereas pharmacological treatment dominated present choices. And yet, future choices tended to emphasize alternative treatment or no treatment at all. Interestingly, the regression analysis added to the CA by revealing that nonpharmacological choices in the past were not predictive of a greater likelihood to choose pharmacological treatment in the present (Figure 4, left graph, red fit), whereas respondents adopting pharmacological treatment in the past were more likely to use non-pharmacological treatment in the present (Figure 4, left graph, blue fit). Likewise, the prediction of future preferences based on present choices indicated that future scenarios were more likely involving non-pharmacological treatments (Figure 4, right graph, red fit) if these were the preferred treatment in the present (clearly involving the "other" category of alternative treatments, Supporting Information S4: Table S3). 

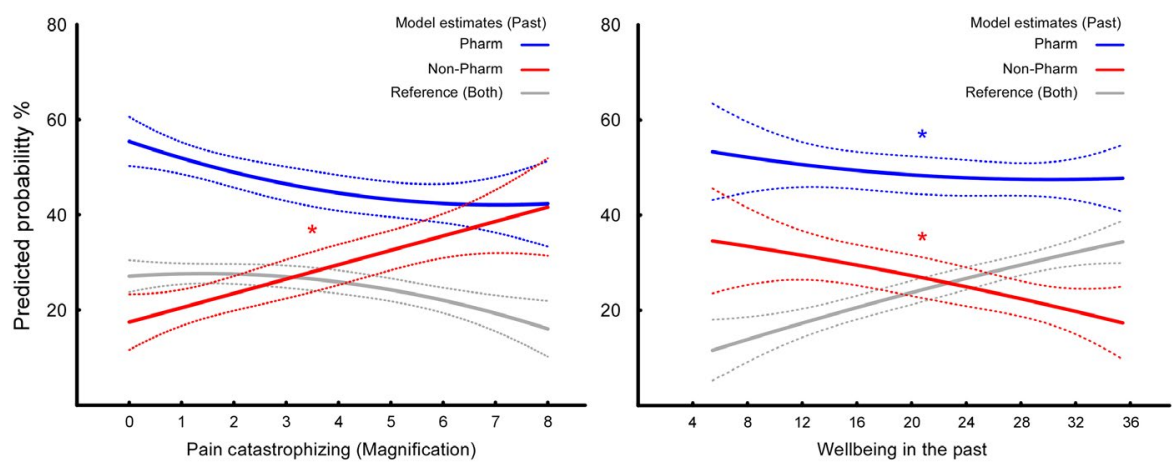

F I G URE 5 Per cent predicted probabilities ( $y$ axis) indicating how likely, compared to the "both" treatment category (grey), respondents were to choose pharmacological (blue) or non-pharmacological (red) treatment in the future based on present choices. The left graph shows the greater likelihood of choosing non-pharmacological treatment in the future if the respondent scored high in pain magnification. The right graph shows the lower likelihood of choosing either pharmacological or non-pharmacological treatment in the present if the respondent reported low well-being in the past (right). Significant predictions are indicated with asterisks $\left({ }^{*} p<.05\right)$. Abbreviations: Pharm, pharmacological; Non-Pharm, non-pharmacological

With regard to the other subjective variables included in the survey, we also found respondents reported that they had significantly lower well-being in the past compared with their current state and future prospect (Table 2). The regression analyses revealed that the respondents reporting higher pain magnification were more likely to choose non-pharmacological treatment in the present (Figure 5, left graph, red fit). In addition, we observed that respondents scoring lower in well-being in the past were more likely to equally opt either for pharmacological or non-pharmacological treatment in the present (Figure 5, right graph).

\section{1 | Attitude to treatment}

This study provides a detailed characterization of a sample of FM individuals representing a diverse FM population. In terms of sample demographics and FM symptoms, our findings are fully compatible with the available epidemiological literature that indicates women outnumber men in the diagnosis of FM. Women are twice as likely to be diagnosed with the FM syndrome compared to men, with the most common age for the onset of the diagnosis between the ages of 25 and 55 (Heidari et al., 2017). Additionally, in line with our study, previous reports indicate that most people diagnosed with FM are Caucasian, middle-aged women reporting pain and fatigue as the most common symptoms (Bennett et al., 2007; Häuser et al., 2012; Jones et al., 2015).

Our respondents opted for pharmacological treatment as well as for non-pharmacological treatment in their past. Significantly, our study provides even more convincing evidence that FM individuals find no satisfying relief in a single treatment therapeutic strategy, and that pharmacological agents do not provide enough therapeutic advantage compared with a non-pharmacological strategy. Crucially, our respondents expressed a preference for less combined treatment in the future compared to the past, as well as a trend in reduction on reliance on pharmacological options over time $(64.9 \%$ in the past, $55 \%$ in the present and $32.8 \%$ in the future). This trend is concomitant with the preference for other treatments not listed in our survey (i.e. OTHER; $27.4 \%, 11.2 \%$ and $32.5 \%$, respectively; see Supporting Information S4: Table S3). However, we also observed an increased number of respondents planning to have no further treatment in the future (14.9\%) compared to the present $(9.1 \%)$ and past (21.8\%). Therefore, we may speculate that while our respondents might have not been able (or wanting) to rely on multiple treatments in the present as much as they had done in the past, they planned to opt again for a diversified treatment strategy in the future, or for no treatment at all, thus outlining an ambivalent pattern of hope and helplessness. Our regression analyses reinforce the presence of this ambivalent pattern by shedding light on the relationship between pharmacological and non-pharmacological treatments: respondents who had previous experience with non-pharmacological treatment showed a preferential trajectory towards non-pharmacological treatment. This preference is in agreement with the EULAR recommendations (Macfarlane et al., 2017) but strikes a contrast with the great reliance on pharmacological treatment overall. Crucially, these are all individuals who were recruited through specialized FM support groups/ organizations, and therefore perhaps educated on FM pathology and treatment. Respondents may have found the treatment less effective than pharmacological treatment or they may have found the psychological treatment to be effective and hence required no further treatment. This last interpretation would reconcile their treatment strategy with the EULAR guidelines, as psychological therapy is recommended as one of the first treatments to newly diagnosed FM patients (Macfarlane et al., 2017). However, it is also possible that FM individuals abandoned psychological therapy over time as its cost may not be viable, especially in the context of a combined treatment strategy. After all, it is well known that FM is experienced as a 
substantial financial burden by patients (Sicras et al., 2009). A similar reasoning can be applied to both physiotherapy and physical therapies which results revealed respondents wanting to return to in the future.

\subsection{Mood, catastrophizing, anxiety and well-being}

The increased frequency of choice for several different treatments in the future, including unlisted alternative treatment, is a finding that hints to the ongoing dissatisfaction of our respondents with their present treatment strategy. This finding, combined with the high degree of mild depression symptoms and high level of state and trait anxiety reported by our respondents, resonates with the most recent recommendations to foster individualized management of FM treatment (Häuser et al., 2017). The FM individuals who participated in our survey are representative of a population affected by a syndrome with intensity ranging from moderate to severe (cumulative 95.3\%). Indeed, almost the entire sample was affected by all the symptoms classically associated with the FM condition such as depression (79.7\%), anxiety (81.8\%), sleep disturbances (98.8\%) and cognitive dysfunctions (91.6\%). This evidence substantiates the notion that FM patients are at risk of developing comorbid psychological conditions (Hudson, Goldenberg, Pope, Keck, \& Schlesinger, 1992) and psychiatric disorders (Fietta, Fietta, \& Manganelli, 2007) compared to the healthy general population.

Additionally, our survey has shown that respondents had a very high tendency to see themselves as helpless when in pain, to ruminate about pain and magnify its threat.

That catastrophizing and depression are risk factors for several disabling long-term health outcomes such as pain severity and physical disability is already an established finding (Edwards, Calahan, Mensing, Smith, \& Haythornthwaite, 2011). Here we report novel evidence showing that pain magnification may be a big factor in determining therapeutic choices in FM individuals, being associated with a higher probability of choosing non-pharmacological approaches in our sample.

The finding that lower well-being in the past was predictive of greater likelihood of the use of a single treatment modality in the present (both pharmacological and non-pharmacological) was mirrored by the finding that higher past well-being was associated with a higher chance of adopting both treatment approaches together (Figure 5, right graph, grey fit). This supports the notion that engaging in more treatments may help patients reducing psychological distress and facilitate a process of acceptance, which seems to be an important psychological dimension promoting well-being in patients with pain (Van Damme, Crombez,
Van Houdenhove, Mariman, \& Michielsen, 2006; Viane et al., 2003).

\section{3 $\quad$ Limitations and future directions}

The present study recruited a self-selected sample of people via support networks so that it was not possible to verify clinician diagnosis. The current study cannot explain why the majority of respondents opted for pharmacological treatment. However, the observation that past/present preference for nonpharmacological treatment leads to higher probability of nonpharmacological treatment preference in the present/future (Figure 4), together with the overall reduction of pharmacological treatment as treatment of choosing over time (Figure 2), hint to the possibility that many respondents were eventually (at least partially) dissatisfied with the pharmacological approach. Because of an excessive amount of missing values among the effectiveness ratings (Supporting Information S5 and S6), we could not provide a reliable analysis of this subjective dimension. Certainly future studies may combine a more robust methodological approach, such as longitudinal data collection (i.e. several temporal estimates), with both subjective and clinical measures of treatment effectiveness in FM individuals. Longitudinal research on patient's preferences may help in identifying better treatments in terms of perceived efficacy at reducing the severity of symptoms.

Another potential limitation of this study originates from the sampling strategy. As we sourced our respondents from FM associations and groups in different nations (see Supporting Information S1), some sociodemographic factors might have injected heterogeneity in respondents' responses. For example, some individuals may have preferred psychological treatment on the basis of insurance coverage provided by insurance companies in some countries (Häuser et al., 2012).

Finally, we failed to introduce aerobic exercise as a default treatment option in the treatment list in our survey design. Aerobic exercise seems to improve quality of life and physical function as well as reducing pain (Bidonde et al., 2017), therefore future studies should explicitly include this category as an item in the treatment list.

\subsection{Conclusions}

This study provides a detailed characterization of a sample of FM individuals representing a diverse FM population. Our findings reveal that pharmacological treatment was the most common preference. However, respondents also reported several treatments in the past and expressed a preference for combined treatment in the future. Importantly, our analyses also indicate that overall the use of both 
pharmacological and non-pharmacological treatments was predictive of later adoption of non-pharmacological treatment but also that the choice of non-pharmacological treatment in the past did not predict preference for pharmacological treatment in the present. Altogether, this ambivalent pattern emphasizes the importance of educating medical professionals on the complexity of this condition as well as supporting patients in their therapeutic choices and disease management within the context of a treatment roadmap (Culpepper, 2012).

\section{ACKNOWLEDGEMENTS}

The authors thank the organizations who helped recruiting respondents who volunteered in the survey, and the respondents themselves. The authors report no conflict of interest.

\section{CONFLICT OF INTEREST}

The authors report no conflict of interest.

\section{AUTHOR CONTRIBUTIONS}

EV conceptualized the study, reviewed the literature, designed the survey, analysed the data, wrote and revised the manuscript and approved the final version to be published. EF conceptualized the study, reviewed the literature, designed the survey, performed a preliminary data analysis, reviewed and approved the final version to be published. SO wrote and critically revised the manuscript and approved the final version to be published. All authors discussed the results and commented on the manuscript.

\section{OR CID}

Elia Valentini (iD https://orcid.org/0000-0003-0259-6824 Eleonora Fetter (D) https://orcid.org/0000-0001-6469-154X Sheina Orbell (D) https://orcid.org/0000-0002-8665-3541

\section{REFERENCES}

Bennett, R. M., Jones, J., Turk, D. C., Russell, I. J., \& Matallana, L. (2007). An internet survey of 2,596 people with fibromyalgia. BMCMusculoskeletal Disorders, 8(1), 27. https://doi.org/10.1186/1471-2474-8-27

Benzecri, J. P. (1992). Correspondence Analysis. New York, NY: Marcel Dekker.

Bernardy, K., Füber, N., Köllner, V., \& Häuser, W. (2010). Efficacy of cognitive-behavioral therapies in fibromyalgia syndrome-A systematic review and metaanalysis of randomized controlled trials. The Journal of Rheumatology, 37(10), 1991-2005. https://doi. org/10.3899/jrheum.100104

Bernardy, K., Klose, P., Welsch, P., \& Häuser, W. (2018). Efficacy, acceptability and safety of cognitive behavioural therapies in fibromyalgia syndrome - A systematic review and meta-analysis of randomized controlled trials. European Journal of Pain, 22(2), 242-260. https://doi.org/10.1002/ejp.1121

Bidonde, J., Busch, A. J., Schachter, C. L., Overend, T. J., Kim, S. Y., Góes, S. M., ... Foulds, H. J. (2017). Aerobic exercise training for adults with fibromyalgia. The. Cochrane Database of Systematic Reviews, 6, CD012700. https://doi.org/10.1002/14651858.CD012700

Branco, J. C., Bannwarth, B., Failde, I., Abello Carbonell, J., Blotman, F., Spaeth, M., ... Matucci-Cerinic, M. (2010). Prevalence of fibromyalgia: A survey in five European countries. Seminars in Arthritis and Rheumatism, 39(6), 448-453. https://doi.org/10.1016/j.semar thrit.2008.12.003

Cording, M., Moore, R. A., Derry, S., \& Wiffen, P. J. (2015). Pregabalin for pain in fibromyalgia in adults. Cochrane Database of Systematic Reviews. https://doi.org/10.1002/14651858.CD011790

Culpepper, L. (2012). A Roadmap for the Management of Fibromyalgia. The Journal of Clinical Psychiatry, https://doi.org/10.4088/ JCP.10118tx3cc

Edwards, R. R., Calahan, C., Mensing, G., Smith, M., \& Haythornthwaite, J. A. (2011). Pain, catastrophizing, and depression in the rheumatic diseases. Nature Reviews Rheumatology, 7(4), 216-224. https://doi. org/10.1038/nrrheum.2011.2

Fietta, P., Fietta, P., \& Manganelli, P. (2007). Fibromyalgia and psychiatric disorders. Acta Biomedica de L'ateneo Parmense. https://doi. org/10.1007/s10741-009-9138-x

Greenacre, M. (2013). Contribution biplots. Journal of Computational and Graphical Statistics, 22(1), 107-122. https://doi. org/10.1080/10618600.2012.702494

Häuser, W., Jung, E., Erbslöh-Möller, B., Gesmann, M., KühnBecker, H., Petermann, F., ... Winkelmann, A. (2012). The German fibromyalgia consumer reports - a cross-sectional survey. BMC Musculoskeletal Disorders, 13(1), 74. https://doi. org/10.1186/1471-2474-13-74

Häuser, W., Perrot, S., Clauw, D. J., \& Fitzcharles, M.-A. (2017). Unravelling fibromyalgia - Steps towards individualized management. The Journal of Pain, 19(2), 125-134. https://doi.org/10.1016/j.jpain.2017.08.009

Häuser, W., Urrútia, G., Tort, S., Üçeyler, N., \& Walitt, B. (2013). Serotonin and noradrenaline reuptake inhibitors (SNRIs) for fibromyalgia syndrome. Cochrane Database of Systematic Reviews. https://doi.org/10.1002/14651858.CD010292

Heidari, F., Afshari, M., \& Moosazadeh, M. (2017). Prevalence of fibromyalgia in general population and patients, a systematic review and meta-analysis. Rheumatology International, 37(9), 1527-1539. https://doi.org/10.1007/s00296-017-3725-2

Hudson, J. I., Goldenberg, D. L., Pope, H. G., Keck, P. E., \& Schlesinger, L. (1992). Comorbidity of fibromyalgia with medical and psychiatric disorders. The American Journal of Medicine, 92(4), 363-367. https://doi.org/10.1016/0002-9343(92)90265-D

Jones, G. T., Atzeni, F., Beasley, M., Flüß, E., Sarzi-Puttini, P., \& Macfarlane, G. J. (2015). The prevalence of fibromyalgia in the general population: A comparison of the American College of Rheumatology 1990, 2010, and modified 2010 classification criteria. Arthritis and Rheumatology, 67(2), 568-575. https://doi.org/10.1002/art.38905

Kroenke, K., \& Spitzer, R. L. (2002). The PHQ-9: A new depression diagnostic and severity measure. Psychiatric Annals, 32(9), 509-515. https://doi.org/10.3928/0048-5713-20020901-06

Lauche, R., Hauser, W., Jung, E., Erbsloh-Moller, B., Gesmann, M., Kuhn-Becker, H., ... Langhorst, J. (2013). Patient-related predictors of treatment satisfaction of patients with fibromyalgia syndrome: Results of a cross-sectional survey. Clinical and Experimental Rheumatology, 31(6 Suppl 79), S34-S40.

Macfarlane, G. J., Kronisch, C., Dean, L. E., Atzeni, F., Häuser, W., Flub, E., ... Jones, G. T. (2017). EULAR revised recommendations for the management of fibromyalgia. Annals of the 
Rheumatic Diseases, 76(2), 318-328. https://doi.org/10.1136/ annrheumdis-2016-209724

Pavot, W., Diener, E., \& Suh, E. M. (1998). The temporal satisfaction ith life scale. Journal of Personality Assessment, 70(2), 350-354. https://doi.org/10.1207/s15327752jpa7002

Robinson, R. L., Kroenke, K., Williams, D. A., Mease, P., Chen, Y., Faries, D., ... Mccarberg, B. (2013). Longitudinal observation of treatment patterns and outcomes for patients with Fibromyalgia: 12-month findings from the REFLECTIONS study. Pain Medicine (USA), 14(9), 1400-1415. https://doi.org/10.1111/pme.12168

Rooij, A. D., Roorda, L. D., Otten, R. H. J., Van Der Leeden, M., Dekker, J., \& Steultjens, M. P. M. (2013). Predictors of multidisciplinary treatment outcome in fibromyalgia:A systematic review. Disability and Rehabilitation, 35(6), 437-449. https://doi.org/10.3109/09638 288.2012.699582

Sicras, A., Rejas, J., Navarro, R., Blanca, M., Morcillo, A., Larios, R., ... Villarroya, C. (2009). Treating patients with fibromyalgia in primary care settings under routine medical practice: A claim database cost and burden of illness study. Arthritis Research \& Therapy, 11(2), R54. https://doi.org/10.1186/ar2673

Sourial, N., Wolfson, C., Zhu, B., Quail, J., Fletcher, J., Karunananthan, S., ... Bergman, H. (2010). Correspondence analysis is a useful tool to uncover the relationships among categorical variables. Journal of Clinical Epidemiology, 63(6), 638-646. https://doi.org/10.1016/j. jclinepi.2009.08.008

Spielberger, C. D., Gorsuch, R. L., Lushene, R., Vagg, P. R., Jacobs, G. A., Lushene, P. R., ... Jacobs, A. G. (1983). Manual for the State-Trait Anxiety Inventory. (C. P. Press., Ed.). https://doi. org/10.1007/978-1-4419-9893-4

Sullivan, M., Bishop, S., \& Pivik, J. (1995). The pain catastrophizing scale: Development and validation. Psychological Assessment, 7(4), 524-532. https://doi.org/10.1037/1040-3590.7.4.524

Teo, M., Mohan, B., \& Oelke, N. D. (2017). Developing and Implementing a Community-Based Model of Care for Fibromyalgia: A Feasibility Study. Pain Research and Management, 2017, 1-9. https://doi.org/10.1155/2017/4521389

Treede, R.-D., Rief, W., Barke, A., Aziz, Q., Bennett, M. I., Benoliel, R., ... Wang, S.-J. (2019). Chronic pain as a symptom or a disease: The IASP classification of chronic pain for the international classification of diseases (ICD-11). Pain, 160(1), 19-27. https://doi. org/10.1097/j.pain.0000000000001384

Turk, D. C., Dworkin, R. H., Revicki, D., Harding, G., Burke, L. B., Cella, D., ... Rappaport, B. A. (2008). Identifying important outcome domains for chronic pain clinical trials: An IMMPACT survey of people with pain. Pain, 137(2), 276-285. https://doi. org/10.1016/j.pain.2007.09.002
Turk, D. C., Okifuji, A., Sinclair, J. D., \& Starz, T. W. (1998). Interdisciplinary treatment for fibromyalgia syndrome: Clinical and statistical significance. Arthritis and Rheumatism, 11(3), 186-195. https://doi.org/10.1002/art.1790110306

Uceyler, N., Hauser, W., \& Sommer, C. (2011). Systematic review with meta-analysis: Cytokines in fibromyalgia syndrome. BMC Musculoskeletal Disorders, 12(1), 245-245. https://doi. org/10.1186/1471-2474-12-245

Van Damme, S., Crombez, G., Van Houdenhove, B., Mariman, A., \& Michielsen, W. (2006). Well-being in patients with chronic fatigue syndrome: The role of acceptance. Journal of Psychosomatic Research, 61(5), 595-599. https://doi.org/10.1016/j.jpsyc hores.2006.04.015

Van Den Houte, M., Luyckx, K., Van Oudenhove, L., Bogaerts, K., Van Diest, I., De Bie, J., \& Van den Bergh, O. (2017). Differentiating progress in a clinical group of fibromyalgia patients during and following a multicomponent treatment program. Journal of Psychosomatic Research, 98, 47-54. https://doi.org/10.1016/j.jpsyc hores.2017.05.004

Viane, I., Crombez, G., Eccleston, C., Poppe, C., Devulder, J., Van Houdenhove, B., \& De Corte, W. (2003). Acceptance of pain is an independent predictor of mental well-being in patients with chronic pain: Empirical evidence and reappraisal. Pain, 106(1), 65-72. https://doi.org/10.1016/S0304-3959(03)00291-4

Wolfe, F., Clauw, D. J., Fitzcharles, M. A., Goldenberg, D. L., Häuser, W., Katz, R. L., ... Walitt, B. (2016). 2016 Revisions to the 2010/2011 fibromyalgia diagnostic criteria. Seminars in Arthritis and Rheumatism, 46(3), 319-329. https://doi.org/10.1016/j.semar thrit.2016.08.012

Wolfe, F., Clauw, D. J., Fitzcharles, M. A., Goldenberg, D. L., Katz, R. S., Mease, P., ... Yunus, M. B. (2010). The American College of Rheumatology preliminary diagnostic criteria for fibromyalgia and measurement of symptom severity. Arthritis Care and Research, 62(5), 600-610. https://doi.org/10.1002/acr.20140

\section{SUPPORTING INFORMATION}

Additional supporting information may be found online in the Supporting Information section.

How to cite this article: Valentini E, Fetter E, Orbell

S. Treatment preferences in fibromyalgia patients: A cross-sectional web-based survey. Eur J Pain. 2020;00:1-11. https://doi.org/10.1002/ejp.1570 